\title{
The edge bending wave on a plate reinforced by a beam
}

\author{
Ahmed SM Alzaidi, ${ }^{1}$ Julius Kaplunov, ${ }^{1}$ and Ludmila Prikazchikova ${ }^{1,}$ a) \\ School of Computing and Mathematics, Keele University, Keele, Staffordshire, \\ ST5 5BG, United Kingdom
}

(Dated: 12 July 2019)

The edge bending wave on a thin isotropic semi-infinite plate reinforced by a beam is considered within the framework of the classical plate and beam theories. The boundary conditions at the plate edge incorporate both dynamic bending and twisting of the beam. A dispersion relation is derived along with its long-wave approximation. The effect of the problem parameters on the cut-offs of the wave in question is studied asymptotically. The obtained results are compared with calculations for the reinforcement in the form of a strip plate.

a)1.prikazchikova@keele.ac.uk 


\section{INTRODUCTION}

Thin elastic structures in the shape of a plate reinforced by a beam have various important applications in naval, civil and aerospace engineering, see ${ }^{1-3}$. Static and dynamic behaviour of stiffened plates was intensively studied in numerous publications within the framework of the classical bending theories for plates and beams also taking into consideration beam torsion, see e.g. ${ }^{4-8}$. At the same time, to the best of authors' knowledge edge waves in stiffened plates have only been analysed in two papers ${ }^{9,10}$, dealing with a semi-infinite strip with simply supported sides. Bending vibrations of an elastic strip were earlier investigated in various setups, e.g. see ${ }^{11,12}$. We also mention the recent authors' contribution $^{13}$ treating a semi-infinite plate reinforced by a strip plate along the edge.

The edge bending wave on an elastic plate has received much attention since long ago, taking into consideration anisotropy, vertical inhomogeneity, contact with elastic foundations, and three dimensional dynamic phenomena, e.g. see the general reference papers ${ }^{14,15}$, and also more recent publications ${ }^{16,17}$. In contrast to the Rayleigh wave on an elastic half space, the plate edge bending wave demonstrates dispersion governed by a specialised parabolicelliptic model ${ }^{18}$.

This note is concerned with qualitative analysis of bending vibrations localised along the edge of a semi-infinite plate, stiffened by a beam. Dispersion relation is derived together with its long-wave asymptotic approximations. At the leading order the latter coincides with the dispersion relation for the plate bending wave on a free edge ${ }^{19}$. Next order solution reveals the influence of stiffening on the edge wave localisation. Comparison of the dispersion 
relation for a plate reinforced by a beam with a narrow rectangular cross-section and for a plate reinforced by a strip plate is demonstrated, justifying the adapted 'plate-beam' formulation.

The effect of material and geometric parameters on edge wave localisation is investigated. A special focus is on the asymptotic evaluation of the cut-offs of the studied edge wave which have been earlier discovered in $^{9,10}$. The possibility of cut-offs over the range of validity of the adapted classical structure theories is addressed.

\section{STATEMENT OF THE PROBLEM}

Consider a thin isotropic elastic plate stiffened by an elastic beam along the edge. The Cartesian coordinate system is chosen in such a way that $x$ and $y$ are in the midplane of the plate with $x$ going along the interface, see Figure 1.

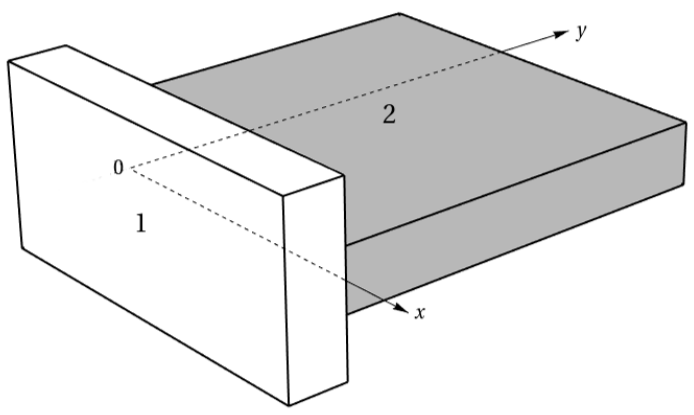

FIG. 1. Plate reinforced by a beam 
The equation of motion for the midplane deflection $w_{2}$ in the classical theory for plate bending is

$$
D_{2}\left(\frac{\partial^{4} w_{2}}{\partial x^{4}}+2 \frac{\partial^{4} w_{2}}{\partial x^{2} \partial y^{2}}+\frac{\partial^{4} w_{2}}{\partial y^{4}}\right)+2 \rho_{2} h \frac{\partial^{2} w_{2}}{\partial t^{2}}=0
$$

where $D_{2}$ is bending stiffness of the plate, $h$ is half thickness of the plate, and $t$ is time. Also, in what follows $\rho_{j}$ are mass densities, $E_{j}$ are Young's moduli, $G_{j}$ are shear moduli, $\nu_{j}$ are Poisson's ratios, $j=1,2$. Indexes 1 and 2 correspond to the beam and plate, respectively.

The boundary conditions for the plate edge $y=0$ maybe obtained by considering the beam flexure and twisting, see for example ${ }^{5}$, resulting in

$$
\begin{aligned}
& E_{1} I_{y} \frac{\partial^{4} w_{2}}{\partial x^{4}}+\rho_{1} A \frac{\partial^{2} w_{2}}{\partial t^{2}}=-D_{2}\left(\frac{\partial^{3} w_{2}}{\partial y^{3}}+\left(2-\nu_{2}\right) \frac{\partial^{3} w_{2}}{\partial x^{2} \partial y}\right) \\
& G_{1} J_{t} \frac{\partial^{3} w_{2}}{\partial x^{2} \partial y}-\rho_{1} J \frac{\partial^{3} w_{2}}{\partial t^{2} \partial y}=-D_{2}\left(\frac{\partial^{2} w_{2}}{\partial y^{2}}+\nu_{2} \frac{\partial^{2} w_{2}}{\partial x^{2}}\right)
\end{aligned}
$$

where $I_{y}$ and $J$ are the area and polar moments of inertia of the beam's cross section, $J_{t}$ is the torsional constant, and $A$ is the area of the beam's cross section.

\section{DISPERSION RELATION}

The solution of the equation (1) is sought for in the form of a travelling harmonic wave as

$$
w_{2}(x, y, t)=w_{2}(y) e^{i(k x-\omega t)},
$$

where $\omega$ is frequency, and $k$ is wave number. Substituting (3) into (1), we arrive for the edge wave at

$$
w_{2}(y)=C_{1} e^{-k \lambda_{1} y}+C_{2} e^{-k \lambda_{2} y},
$$


where $C_{1}$ and $C_{2}$ are arbitrary constants, and

$$
\lambda_{1}=\sqrt{1+\gamma_{2}}, \quad \lambda_{2}=\sqrt{1-\gamma_{2}}, \quad \gamma_{2}=\frac{\omega}{k^{2}} \sqrt{\frac{2 \rho_{2} h}{D_{2}}} .
$$

Now, on substituting (3) into the boundary conditions (2) we arrive at the $2 \times 2$ set of linear equations, leading to the general exact dispersion relation

$$
\begin{aligned}
& \left(\lambda_{1} \lambda_{2}+1\right)^{2}-\nu_{2}\left(\lambda_{1}+\lambda_{2}\right)^{2}-\left(1-\nu_{2}\right)^{2} \\
& -\left(\lambda_{1}+\lambda_{2}\right)\left(\alpha_{1} \gamma_{2}^{2} \rho-\beta_{2} \lambda_{1} \lambda_{2}-\beta_{1}\right) \delta_{h} \\
& -\beta_{2}\left(\alpha_{1} \gamma_{2}^{2} \rho-\beta_{1}\right) \delta_{h}^{2}-\alpha_{2} \gamma_{2}^{2} \rho \lambda_{1} \lambda_{2}\left(\lambda_{1}+\lambda_{2}\right) \delta_{h}^{3} \\
& +\alpha_{2} \gamma_{2}^{2} \rho\left(\alpha_{1} \gamma_{2}^{2} \rho-\beta_{1}\right) \delta_{h}^{4}=0,
\end{aligned}
$$

where

$$
\alpha_{1}=\frac{A}{2 h^{2}}, \quad \alpha_{2}=\frac{J}{2 h^{4}}, \quad \beta_{1}=\frac{E_{1} I_{y}}{h D_{2}}, \quad \beta_{2}=\frac{G_{1} J_{t}}{h D_{2}},
$$

and $\delta_{h}=k h, \rho=\frac{\rho_{1}}{\rho_{2}}$.

Setting $\delta_{h}=0$ in (4) and returning back to original variables we get the well known relation for a free plate edge, see e.g. ${ }^{19}$

$$
D_{2} k^{4} c^{4}=2 \rho_{2} h \omega^{2}
$$

where

$$
c=\left[\left(1-\nu_{2}\right)\left(3 \nu_{2}-1+2 \sqrt{2 \nu_{2}^{2}-2 \nu_{2}+1}\right)\right]^{1 / 4} .
$$

Let us next introduce a new unknown function by

$$
\phi=\frac{\sqrt{1-\gamma_{2}^{2}}}{\nu_{2}^{2}},
$$

corresponding to the appropriately normalised attenuation rate which is not sensitive to the value of a Poison's ratio. This is seemingly the most relevant characteristic of slowly 
decaying edge bending waves. Hence, equation (4) can be re-written as

$$
\begin{aligned}
& \left(1+\nu_{2}^{2} \phi\right)^{2}-2 \nu_{2}\left(1+\nu_{2}^{2} \phi\right)-\left(1-\nu_{2}\right)^{2} \\
& -\sqrt{2\left(1+\nu_{2}^{2} \phi\right)}\left(\alpha_{1} \rho\left(1-\nu_{2}^{4} \phi^{2}\right)-\beta_{2} \nu_{2}^{2} \phi-\beta_{1}\right) \delta_{h} \\
& -\beta_{2}\left(\alpha_{1} \rho\left(1-\nu_{2}^{4} \phi^{2}\right)-\beta_{1}\right) \delta_{h}^{2} \\
& -\alpha_{2} \nu_{2}^{2} \rho\left(1-\nu_{2}^{4} \phi^{2}\right) \phi \sqrt{2\left(1+\nu_{2}^{2} \phi\right)} \delta_{h}^{3} \\
& +\alpha_{2} \rho\left(\alpha_{1} \rho\left(1-\nu_{2}^{4} \phi^{2}\right)-\beta_{1}\right)\left(1-\nu_{2}^{4} \phi^{2}\right) \delta_{h}^{4}=0,
\end{aligned}
$$

61

and

$$
\phi_{1}=\frac{\left(\left(1-\nu_{2}^{4} \phi_{0}^{2}\right) \rho \alpha_{1}-\beta_{1}-\beta_{2} \nu_{2}^{2} \phi_{0}\right) \sqrt{2\left(1+\nu_{2}^{2} \phi_{0}\right)}}{2 \nu_{2}^{4} \phi_{0}-2\left(\nu_{2}-1\right) \nu_{2}^{2}} .
$$




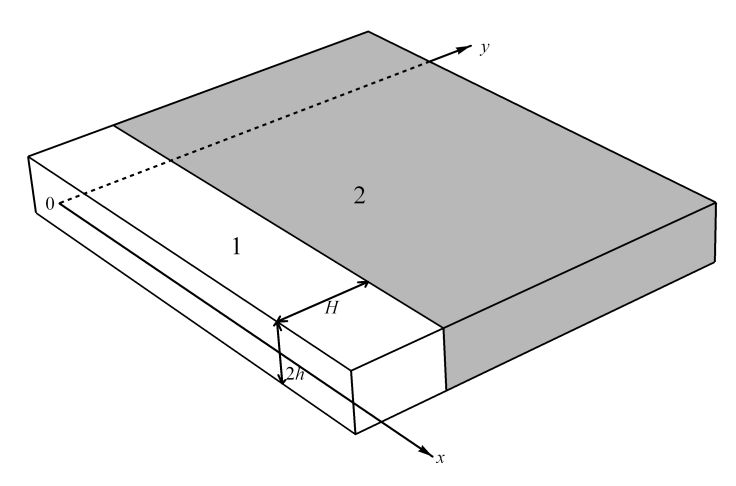

FIG. 2. Plate reinforced by a strip plate on parameter $\beta_{2}$, expressing the effect of torsional rigidity.

\section{EXAMPLE} substituting 1 instead of 2 in all the suffices.

It is worth noting that (8) and (9)-(11) do not contain the parameter $\alpha_{2}$ involving rotation inertia of the beam. This is in line with the asymptotic analysis of a similar problem for the edge reinforcement in the form of a plate strip in ${ }^{13}$. In addition, (8) also does not depend

In this section we present the results of numerical comparison of the dispersion curves for a plate reinforced by a beam and a composite 'plate-plate' structure, in order to validate the 'plate-beam' model in the previous section. To this end, consider bending of a semi-infinite Kirchhoff plate reinforced by a strip plate along the edge as shown in Figure 2, assuming that for the strip plate $H \gg h$. For the latter, the equation of motion follows from (1) by

73 Traction free boundary conditions on the edge $y=0$ are given by

$$
\frac{\partial^{2} w_{1}}{\partial y^{2}}+\nu_{1} \frac{\partial^{2} w_{1}}{\partial x^{2}}=0, \quad \frac{\partial^{3} w_{1}}{\partial y^{3}}+\left(2-\nu_{1}\right) \frac{\partial^{3} w_{1}}{\partial x^{2} \partial y}=0
$$


The continuity conditions at $y=H$ are

$$
\begin{aligned}
& w_{1}=w_{2} \\
& \frac{\partial w_{1}}{\partial y}=\frac{\partial w_{2}}{\partial y} \\
& D_{1}\left(\frac{\partial^{2} w_{1}}{\partial y^{2}}+\nu_{1} \frac{\partial^{2} w_{1}}{\partial x^{2}}\right)=D_{2}\left(\frac{\partial^{2} w_{2}}{\partial y^{2}}+\nu_{2} \frac{\partial^{2} w_{2}}{\partial x^{2}}\right), \\
& D_{1}\left(\frac{\partial^{3} w_{1}}{\partial y^{3}}+\left(2-\nu_{1}\right) \frac{\partial^{3} w_{1}}{\partial x^{2} \partial y}\right)=D_{2}\left(\frac{\partial^{3} w_{2}}{\partial y^{3}}+\left(2-\nu_{2}\right) \frac{\partial^{3} w_{2}}{\partial x^{2} \partial y}\right) .
\end{aligned}
$$

74 The related dispersion equation is

$$
\operatorname{det} \mathbf{M}=0 \text {, }
$$

with the non-zero components of $6 \times 6$ matrix $\mathbf{M}$ given in Appendix, where the notation

$$
D=\frac{D_{1}}{D_{2}}
$$

75 is introduced.

For a plate reinforced by a beam with a narrow rectangular cross section the quantities $I_{y}, J, J_{t}$, and $A$ in (2) are defined as

$$
I_{y}=\frac{2}{3} h^{3} H, \quad J=\frac{1}{6} h H^{3}, \quad J_{t}=\frac{8}{3} h^{3} H, \quad A=2 h H .
$$

Taking into account the relations

$$
D_{j}=\frac{2 E_{j} h^{3}}{3\left(1-\nu_{j}^{2}\right)}, \quad G_{j}=\frac{E_{j}}{2\left(1+\nu_{j}\right)}, \quad j=1,2
$$

we have

$$
\alpha_{1}=\eta, \quad \alpha_{2}=\frac{1}{12} \eta^{3}, \quad \beta_{1}=D\left(1-\nu_{1}^{2}\right) \eta, \quad \beta_{2}=2 D\left(1-\nu_{1}\right) \eta,
$$


where $\eta=H / h$. Substituting the above formulae into (6) we obtain dispersion equation

$$
\begin{aligned}
& \left(1+\nu_{2}^{2} \phi\right)^{2}-2 \nu_{2}\left(1+\nu_{2}^{2} \phi\right)-\left(1-\nu_{2}\right)^{2}-\sqrt{2\left(1+\nu_{2}^{2} \phi\right)} \times \\
& \left(\rho\left(1-\nu_{2}^{4} \phi^{2}\right)-D\left(1-\nu_{1}\right)\left(1+\nu_{1}+2 \nu_{2}^{2} \phi\right)\right) \delta_{H} \\
& -2 D\left(1-\nu_{1}\right)\left(\rho\left(1-\nu_{2}^{4} \phi^{2}\right)-D\left(1-\nu_{1}^{2}\right)\right) \delta_{H}^{2} \\
& -\frac{1}{12}\left(1-\nu_{2}^{4} \phi^{2}\right) \nu_{2}^{2} \rho \phi \sqrt{2\left(1+\nu_{2}^{2} \phi\right)} \delta_{H}^{3} \\
& +\frac{1}{12}\left(1-\nu_{2}^{4} \phi^{2}\right) \rho\left(\rho\left(1-\nu_{2}^{4} \phi^{2}\right)-D\left(1-\nu_{1}^{2}\right)\right) \delta_{H}^{4}=0,
\end{aligned}
$$

77

where $\delta_{H}=k H \ll 1$. Now, the cut-off at leading order is given by the formula

$$
\delta_{H}^{*} \approx \frac{\nu_{2}^{2}}{\sqrt{2}\left(D\left(1-\nu_{1}^{2}\right)-\rho\right)},
$$

which readily follows from (8) and is valid provided that $\nu_{2}^{2} \ll 1$ and $D\left(1-\nu_{1}^{2}\right)>\rho$.

Also, the asymptotic expansion for $\phi$, analogous to (9), becomes

$$
\phi=\widetilde{\phi}_{0}+\widetilde{\phi}_{1} \delta_{H}+\ldots
$$

where $\widetilde{\phi}_{0}=\phi_{0}$ and

$$
\begin{aligned}
\widetilde{\phi}_{1}= & \sqrt{2\left(1+\nu_{2}^{2} \phi_{0}\right)} \times \\
& \frac{\left(\rho\left(1-\nu_{2}^{4} \phi_{0}^{2}\right)-D\left(1-\nu_{1}\right)\left(1+\nu_{1}+\nu_{2}^{2} \phi_{0}\right)\right)}{2 \nu_{2}^{2}\left(1-\nu_{2}+\nu_{2}^{2} \phi_{0}\right)} .
\end{aligned}
$$

In Figures 3 and 4 the function $\phi$ is plotted against dimensionless wave number $\delta_{H}$. In these figures the dispersion curves for a plate reinforced by a beam (15) and by a strip plate (13) are plotted together with those corresponding to the two term asymptotic approximations (17). Numerical examples are presented for $\nu_{1}=0.31$ and $\nu_{2}=0.35$.

As might be expected, both beam approximation (15) and its two-term asymptotics (17) are robust only over the long wave range $\left(\delta_{H} \ll 1\right)$, see the curves for $D=2.3$ in Figure 


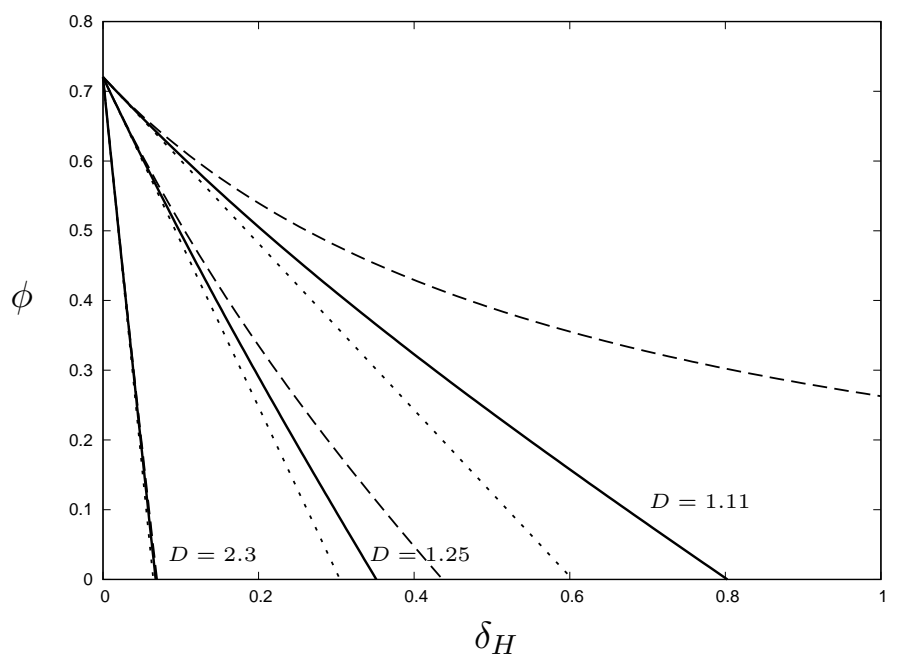

FIG. 3. Comparison of dispersion relations (13) (solid line), (15) (dashed line) and asymptotic expansion (17) (dotted line) for $\rho=1.0$ and $D=2.3,1.25,1.11$.

3 and $\rho=0.2$ in Figure 4, for which the asymptotic formulae (16) gives $\delta_{H}^{*}=0.08$ and $\delta_{H}^{*}=0.12$, respectively. Outside the long wave range, the deviation between the results for plate and beam edge reinforcement become more substantial. In particular, as follows from formula (7) with (14) the beam reinforcement does not assume a cut-off under the condition $D\left(1-\nu_{1}^{2}\right)-\rho=0$, which is satisfied for the curves corresponding to $D=1.11$ in Figure 3 and $\rho=0.9$ in Figure 4. At the same time, for both of these scenario the strip plate reinforcement predicts cut-offs at $\delta_{H}^{*} \sim 1$.

\section{CONCLUSION}

We studied the edge wave problem for a semi-infinite plate reinforced by a beam taking into account both bending and twisting vibrations of the beam. The explicit asymptotic formulae for the cut-offs of the edge waves are presented. The validity of the chosen approximate formulation starting from the classical plate and beam theories is also addressed. Dis- 


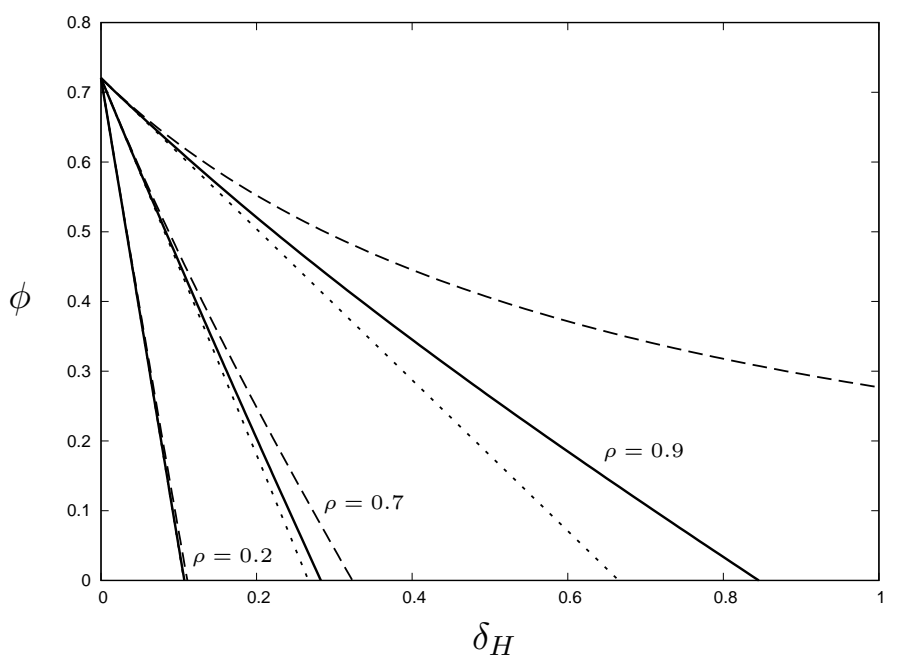

FIG. 4. Comparison of dispersion relations (13) (solid line), (15) (dashed line) and asymptotic expansion (17) (dotted line) for $D=1.0$ and $\rho=0.2,0.7,0.9$.

persion relation is obtained and long-wave approximation is derived. The numerical results are validated by comparison with the more general dispersion relation for a reinforcement in the form of a strip plate, which is also treated on the basis of the 2D Kirchhoff theory. The developed framework may be extended to more general setups including anisotropic structures as well as more elaborated structure models, e.g. see ${ }^{14,20}$. 


\section{APPENDIX}

The entries of the matrix $M$ in (13) are given by

$$
\begin{aligned}
& M_{11}=M_{12}=\lambda_{11}^{2}-\nu_{1}, \quad M_{13}=M_{14}=\lambda_{21}^{2}-\nu_{1} \\
& M_{21}=-M_{22}=\lambda_{11}\left(\lambda_{11}^{2}+\nu_{1}-2\right), \\
& M_{23}=-M_{24}=\lambda_{21}\left(\lambda_{21}^{2}+\nu_{1}-2\right), \\
& M_{31}=\lambda_{11} e^{\lambda_{11} \delta_{H}}, \quad M_{32}=-\lambda_{11} e^{-\lambda_{11} \delta_{H}}, \\
& M_{33}=\lambda_{21} e^{\lambda_{21} \delta_{H}}, \quad M_{34}=-\lambda_{21} e^{-\lambda_{21} \delta_{H}}, \\
& M_{35}=\lambda_{12} e^{-\lambda_{12} \delta_{H}}, \quad M_{36}=\lambda_{22} e^{-\lambda_{22} \delta_{H}}, \\
& M_{41}=e^{\lambda_{11} \delta_{H}}, \quad M_{42}=e^{-\lambda_{11} \delta_{H}}, \quad M_{43}=e^{\lambda_{21} \delta_{H}}, \\
& M_{44}=e^{-\lambda_{21} \delta_{H}}, \quad M_{45}=-e^{-\lambda_{12} \delta_{H}}, \quad M_{46}=-e^{-\lambda_{22} \delta_{H}}, \\
& M_{51}=D\left(\lambda_{11}^{2}-\nu_{1}\right) e^{\lambda_{11} \delta_{H}}, \quad M_{52}=D\left(\lambda_{11}^{2}-\nu_{1}\right) e^{-\lambda_{11} \delta_{H}}, \\
& M_{53}=D\left(\lambda_{21}^{2}-\nu_{1}\right) e^{\lambda_{21} \delta_{H}}, \quad M_{54}=D\left(\lambda_{21}^{2}-\nu_{1}\right) e^{-\lambda_{21} \delta_{H}}, \\
& M_{55}=-\left(\lambda_{12}^{2}-\nu_{2}\right) e^{-\lambda_{12} \delta_{H}}, \quad M_{56}=-\left(\lambda_{22}^{2}-\nu_{2}\right) e^{-\lambda_{22} \delta_{H}}, \\
& M_{61}=D \lambda_{11}\left(\lambda_{11}^{2}+\nu_{1}-2\right) e^{\lambda_{11} \delta_{H}}, \\
& M_{62}=-D \lambda_{11}\left(\lambda_{11}^{2}+\nu_{1}-2\right) e^{-\lambda_{11} \delta_{H}}, \\
& M_{63}=D \lambda_{21}\left(\lambda_{21}^{2}+\nu_{1}-2\right) e^{\lambda_{21} \delta_{H}}, \\
& M_{64}=-D \lambda_{21}\left(\lambda_{21}^{2}+\nu_{1}-2\right) e^{-\lambda_{21} \delta_{H}}, \\
& M_{65}=\lambda_{12}\left(\lambda_{12}^{2}+\nu_{2}-2\right) e^{-\lambda_{12} \delta_{H}}, \\
& M_{66}=\lambda_{22}\left(\lambda_{22}^{2}+\nu_{2}-2\right) e^{-\lambda_{22} \delta_{H}},
\end{aligned}
$$


where

$$
\lambda_{1 j}=\sqrt{1+\gamma_{j}}, \quad \lambda_{2 j}=\sqrt{1-\gamma_{j}},
$$

and

$$
\gamma_{j}=\frac{\omega}{k^{2}} \sqrt{\frac{2 \rho_{j} h}{D_{j}}}, \quad j=1,2 .
$$

\section{ACKNOWLEDGMENT}

This work was supported by the grant J2-9224 from the Slovenian Research Agency.

\section{REFERENCES}

${ }^{1}$ J.Farkas, and K.Jármai, "Optimum design of steel structures," Heidelberg: Springer (2013).

${ }^{2}$ S.C.Misra, "Design principles of ships and marine structures," CRC Press (2015).

${ }^{3}$ H.Singh, "Laterally loaded reinforced concrete stiffened plates: analytical investigations,"

Pract. Period. Struct. Des. Construct. 17(1), 21-29 (2011).

${ }^{4}$ I.Elishakoff, and A.Sternberg, "Vibration of rectangular plates with edge-beams," Acta Mech. 36(3-4), 195-212 (1980).

${ }^{5}$ D.A.Pape, and A.J.Fox, "Deflection solutions for edge stiffened plates," In Proceedings of the 2006 IJME-INTERTECH Conference, 203-091.

${ }^{6}$ E.J.Sapountzakis, and J. T.Katsikadelis, "Analysis of plates reinforced with beams," Comput. Mech. 26(1), 66-74 (2000). 
${ }^{7}$ Y.K.Lin, "Probabilistic theory of structural dynamics," New York, McGraw-Hill book Co (1967).

${ }^{8}$ L.Dozio, and M.Ricciardi, "Free vibration analysis of ribbed plates by a combined analytical-numerical method," J. Sound Vib. 319(1-2), 681-697 (2009).

${ }^{9}$ M.Belubekyan, K.Ghazaryan, P.Marzocca, and C.Cormier, "Localized bending waves in a rib-reinforced elastic orthotropic plate," J. Appl. Mech. 74(1), 169-171 (2007).

${ }^{10}$ A.Milanese, P.Marzocca, M.Belubekyan, and K.Ghazaryan, "Effect of the stiffness and inertia of a rib reinforcement on localized bending waves in semi-infinite strips," Int. J. Solids Struct. 46(10), 2126-2135 (2009).

${ }^{11}$ Y.K.Konenkov, "On normal modes of flexural waves in a plate," Sov. Acoust. Phys. 6(1), 57-64 (1960).

${ }^{12}$ J.D.Kaplunov, L.Y.Kossovich, and M.V.Wilde, "Free localized vibrations of a semi-infinite cylindrical shell," J. Acoust. Soc. Am. 107(3), 1383-1393 (2000).

${ }^{13}$ A.S.Alzaidi, J.Kaplunov, and L.Prikazchikova, "Elastic bending wave on the edge of a semi-infinite plate reinforced by a strip plate," Math. Mech. Solids, 1081286519840687 (2019).

${ }^{14}$ A.N.Norris, V.V.Krylov, and I.D.Abrahams, "Flexural edge waves and Comments on "A new bending wave solution for the classical plate equation [J. Acoust. Soc. Am. 104, 22202222 (1998)]"," J. Acoust. Soc. Am. 107(3), 1781-1784 (2000).

${ }^{15}$ J.B.Lawrie, and J.Kaplunov, "Edge waves and resonance on elastic structures: An overview," Math. Mech. Solids 17(1), 4-16 (2012). 
${ }^{16}$ J.Kaplunov, D.A.Prikazchikov, G.A.Rogerson, and M.I.Lashab, "The edge wave on an elastically supported Kirchhoff plate," J. Acoust. Soc. Am. 136(4), 1487-1490 (2014).

${ }^{17}$ J.Kaplunov, and A.Nobili, "The edge waves on a Kirchhoff plate bilaterally supported by a two-parameter elastic foundation," J. Vib. Control 23(12), 2014-2022 (2017).

${ }^{18}$ J.Kaplunov, D.A.Prikazchikov, and G.A.Rogerson, "Edge bending wave on a thin elastic plate resting on a Winkler foundation," Proc. Royal Soc. A 472(2190), 20160178 (2016).

${ }^{19}$ Y.K.Konenkov, "A Rayleigh-type flexural wave," Sov. Phys. Acoust. 6, 122-123 (1960).

${ }^{20}$ D.D.Zakharov, "Analysis of the acoustical edge flexural mode in a plate using refined asymptotics," J. Acoust. Soc. Am. 116(2), 872-878 (2004). 